\title{
Adaptação transcultural: tradução e validação de conteúdo da versão brasileira do Commitment Exercise Scale
}

\author{
Transcultural adaptation: translation and content validation of the Commitment Exercise Scale to the Brazilian \\ language
}

\author{
Paula Costa Teixeira1,2, Norman Hearst³ ${ }^{3}$, Sandra Marcela Mahecha Matsudo², Táki Athanássios Cordás1, \\ Maria Aparecida CONTI ${ }^{1}$
}

1 Coordenador-geral do Ambulatório de Bulimia e Transtornos Alimentares (Ambulim) do Instituto de Psiquiatria do Hospital das Clínicas da FMUSP, professor-colaborador do Departamento de Psiquiatria da FMUSP, professor do Programa de Pós-Graduação do Departamento de Psiquiatria da FMUSP e do Programa de Neurociências e Comportamento do Instituto de Psicologia da USP. 2 Centro de Estudos do Laboratório de Aptidão Física de São Caetano do Sul (Celafiscs).

${ }^{3}$ Departamento de Medicina Comunitária e Familiar e Departamento de Epidemiologia e Bioestatística da Universidade da Califórnia, Estados Unidos.

Recebido: 17/12/2009 - Aceito: 30/3/2010

\begin{abstract}
Resumo
Contexto: O Commitment Exercise Scale (CES) avalia o comprometimento, o comportamento e a atitude de pacientes com transtornos alimentares em relação ao exercício físico. Objetivo: Tradução e adaptação transcultural do CES para o idioma português (BRA), validação de conteúdo e análise da consistência interna. Métodos: Envolveu cinco etapas: (1) tradução; (2) retradução; (3) revisão técnica e avaliação semântica; (4) validação de conteúdo por profissionais da área - juízes; (5) avaliação do instrumento por uma amostra de estudantes, por meio da avaliação do grau de compreensão e análise da consistência interna do instrumento pelo coeficiente $\alpha$ de Cronbach. Resultados: A escala foi traduzida e adaptada, sendo considerada de fácil compreensão (grau de compreensão de 4,3 a 4,9), e demonstrou valores do coeficiente aceitáveis ( $\alpha$ de Cronbach: 0,60 a 0,79). Conclusão: A partir do processo de adaptação transcultural, os resultados satisfatórios possibilitam a recomendação da versão brasileira do CES. São necessárias análises de validação de construto, fatorial e de reprodutibilidade.
\end{abstract}

Teixeira PC, et al. / Rev Psiq Clín. 2011;38(1):24-28

Palavras-chave: Tradução, escalas, estudos de validação, transtornos alimentares, exercício físico.

\begin{abstract}
Background: The Exercise Commitment Scale (CES) evaluates commitment, attitude and behavior regarding exercise in eating disorders patients. Objective: Translation and cultural adaptation of the CES for Portuguese (BRA), content validation and analysis of internal consistency. Methods: This study involved five steps: (1) translation, (2) re-translation, (3) technical review and evaluation semantics, (4) content validation by professionals - judges, (5) assessment of the instrument in a sample of students, by assessing the degree of understanding and analysis of internal consistency by Cronbach's $\alpha$ coefficient. Results: The scale was translated and adapted. It was considered easy to understand (degree of understanding from 4.3 to 4.9 ) and showed acceptable consistency coefficients (Cronbach's $\alpha$ : 0.60 to 0.79 ). Discussion: Satisfactory results of this adaptation process allow the recommendation of the CES Brazilian version. Analysis is needed of construct and factorial validation, and of reproducibility.
\end{abstract}

Teixeira PC, et al. / Rev Psiq Clín. 2011;38(1):24-28

Keywords: Translation, scales, validation studies, eating disorders, exercise.

\section{Introdução}

A associação entre a prática regular de atividade física e a conquista da saúde está evidenciada por meio de estudos epidemiológicos ${ }^{1-4}$. Desde a década de 1970, a atividade física vem sendo alvo de inúmeras pesquisas tendo como uma das metas a avaliação do nível de atividade física na população. $\mathrm{O}$ combate ao sedentarismo, ou seja, a inatividade física, não está relacionado somente com doenças e mortalidade, mas também com o alto custo econômico à sociedade 3,5 .

A atividade física estruturada ou o exercício físico quando praticado regularmente resulta em benefícios físicos e psicológicos ${ }^{4}$. Porém, estudos recentes sugerem que o exercício físico pode ser prejudicial para alguns indivíduos que o praticam de forma excessiva, podendo desenvolver uma relação de dependência ${ }^{6,7}$.

A dependência de exercício físico pode ser definida como um processo em que o indivíduo se sente obrigado a exercitar-se e apresenta sintomas físicos e psicológicos de abstinência quando não pode se exercitar ${ }^{8}$. De Coverley Veale ${ }^{9}$ explica que indivíduos dependentes do exercício físico apresentam sintomas semelhantes aos com dependência de substâncias químicas, como tolerância e abstinência, além de problemas psicossociais ${ }^{6}$.
Diferentes termos são utilizados para determinar esse tipo de prática, por exemplo: exercise addiction - dependência de exercício ${ }^{10}$; negative addiction - dependência negativa ${ }^{11}$; obligatory running obrigação em correr ${ }^{12}$; morbid exercise - exercício mórbido ${ }^{13}$; exercise dependence - dependência de exercício ${ }^{14}$; compulsive running - correr compulsivo ${ }^{13}$; compulsive exercise - exercício compulsivo ${ }^{15}$; excessive exercise - exercício excessivo ${ }^{16}$.

Diante da falta de clareza das definições desses conceitos, inúmeros instrumentos foram desenvolvidos na tentativa de avaliar os sintomas, o que dificulta ainda mais para a comunidade científica formular um consenso acerca da dependência de exercício físico.

Um dos grupos populacionais que apresentam sintomas de dependência de exercício físico é o de indivíduos com transtornos alimentares. A anorexia nervosa (AN) e a bulimia nervosa (BN) são os transtornos mais comuns ${ }^{17}$. A AN é caracterizada por perda de peso intensa e intencional devida a dietas rígidas, busca desenfreada pela magreza e grave distorção do aspecto perceptivo da imagem corporal18. Já a BN é caracterizada por grande ingestão de alimentos com sensação de perda de controle, preocupação excessiva com o peso e a imagem corporal, bem como a utilização de métodos 
compensatórios inadequados para controle do peso como vômitos, medicamentos, dietas e exercícios físicos ${ }^{19}$.

Sugere-se que aproximadamente $80 \%$ dos pacientes com diagnóstico de $\mathrm{AN}$ e $55 \%$ dos pacientes com $\mathrm{BN}$ praticam exercício físico de forma inadequada em algum momento de sua história clínica ${ }^{20}$. A prevalência da $\mathrm{AN}$ na população geral é de $1 \%$ e no caso da $\mathrm{BN}$ a prevalência pode chegar a $4 \%^{21}$.

Há evidências que associam a dependência de exercício físico com os transtornos alimentares. Porém, é importante ressaltar que isso não é uma regra. Alguns indivíduos com transtornos alimentares podem apresentar esse sintoma pelo interesse em controlar o peso e/ou a composição corporal ${ }^{13}$. De acordo com De Coverley Veale ${ }^{9}$, a prática de exercício físico em indivíduos com transtornos alimentares pode ser definida como uma dependência secundária. A dependência primária seria referente a uma prática com o único fim de se exercitar, portanto deve ser excluído o diagnóstico de transtorno alimentar.

Para investigar se a prática de exercícios físicos para controlar o peso predizia sintomas de transtornos alimentares, Adkins e Keel ${ }^{22}$ avaliaram o exercício físico em dois aspectos: a quantidade e a qualidade. A quantidade indicava a frequência em dias por semana, a duração da sessão (minutos ou horas) e a intensidade (leve, moderada, vigorosa) da atividade realizada; já a qualidade caracterizava-se pela existência de troca de outras atividades de lazer ou familiares por uma agenda rígida, em que o indivíduo não se permite dias sem exercício físico. Quando isso eventualmente ocorresse por alguma razão, o indivíduo apresentava um quadro com sintomas ansiosos, depressivos e irritabilidade por ter perdido a sessão de exercícios ${ }^{22}$. Indivíduos assim podem ser chamados de compulsivos ou dependentes de exercícios físicos, uma vez que esses critérios aproximam esse fenômeno da síndrome de abstinência encontrado em dependentes químicos.

Entre os instrumentos elaborados para avaliar a relação entre transtornos alimentares e exercício físico, tem-se: Commitment to Running Scale ${ }^{23}$, Obligatory Exercise Questionnaire (OEQ) ${ }^{15}$ e Commitment Exercise Scale (CES) ${ }^{16}$.

A Commitment to Running Scale proposta por Carmack e Marten $^{23}$ foi a primeira escala a investigar a dependência de exercício físico, porém o instrumento avalia apenas o compromisso do indivíduo em correr ${ }^{13}$, o que o torna muito específico, não sendo adequado para avaliar outras formas de dependência de exercício físico ${ }^{6}$.

$\mathrm{O} \mathrm{OEQ}^{15}$ é um questionário que contém 20 itens e avalia o comportamento e a atitude do indivíduo em relação à prática de exercícios físicos. Apesar de ser usado frequentemente e apresentar propriedades psicométricas bem estabelecidas, os autores não fornecem informações detalhadas sobre o constructo ${ }^{8}$.

Um dos instrumentos que têm sido muito utilizados nas pesquisas que investigam a relação entre exercício físico e transtornos alimentares é o CES 16 . Para desenvolvê-lo, Davis et al. ${ }^{16}$ basearam-se nas publicações de estudos de caso de homens e mulheres que tinham o hábito de se exercitar de maneira considerada pelos autores como excessiva e patológica. A escala foi validada respeitando os critérios psicométricos e avalia o grau de aderência do indivíduo ao exercício físico mesmo em condições adversas, cujo exercício pode interferir no aspecto social. Consiste em oito perguntas que avaliam o grau de compromisso do indivíduo com a prática de exercícios físicos. Cada pergunta da escala possui uma linha de $155 \mathrm{~mm}$ com duas opções de resposta, sendo uma no início e outra no final da linha. O indivíduo deve descrever com um ponto na linha qual a sua posição. A distância entre o começo da linha e o ponto marcado consiste na pontuação de cada item ${ }^{16}$. Mond et al. ${ }^{24}$ verificaram uma forte associação entre as questões do CES e os sintomas de transtornos alimentares. Além da sua praticidade de aplicação, sua utilização em estudos futuros permitirá melhor comparação com dados já existentes na comunida científica.

Em virtude da falta de instrumentos validados para esse fim no Brasil, o objetivo do presente estudo é apresentar a adaptação transcultural por meio da tradução, dados de valiadação de conteúdo e consistência interna do CES.

\section{Métodos}

A adaptação transcultural baseou-se nos procedimentos sugeridos por Reichenheim e Moraes ${ }^{25}$ e aplicados por Conti et al.26,27. Dessa forma, foram cumpridas cinco etapas.

Para a primeira etapa, o instrumento original, na versão inglesa, foi traduzido para o idioma português de forma independente, por duas pesquisadoras experientes e fluentes em inglês. Uma versão foi elaborada e em uma segunda etapa o instrumento foi novamente traduzido para o inglês, por um professor nativo de língua inglesa. A segunda etapa consistiu na revisão técnica e avaliação da equivalência semântica, por meio da comparação das três versões existentes (original - inglês, português e versão retrotraduzida inglês), valorizando-se o significado referencial do instrumento, da versão traduzida (português) em relação à versão final (inglês) e o significado geral, na comparação da versão original em relação à versão retrotraduzida (inglês) ${ }^{25}$.

$\mathrm{Na}$ etapa seguinte, o instrumento foi avaliado por especialistas da área de saúde mental (três psicólogos, duas enfermeiras, quatro nutricionistas, três psiquiatras e um educador físico) totalizando 13 profissionais. Foi aplicado um questionário e solicitado, a cada participante, que avaliasse a clareza e o grau de compreensão de cada questão e da instrução da escala, por meio de uma escala verbal-numérica adaptada ${ }^{26,27}$. Para isso, o profissional deveria responder à seguinte questão: "Você entendeu o que foi perguntado?". As respostas eram do tipo escala Likert: 0 - não entendi nada; 1 - entendi só um pouco; 2 - entendi mais ou menos; 3 - entendi quase tudo, mas tive algumas dúvidas; 4 - entendi quase tudo; 5 - entendi perfeitamente e não tenho dúvidas. Foram utilizadas como critério de compreensão insuficiente as respostas $0,1,2$ e 3, conforme sugerido por Conti et al. ${ }^{26,27}$. E caso o especialista não compreendesse a questão ou a linguagem não parecesse adequada, foi solicitado que sugerisse alterações, justificando os motivos. Os profissionais também assinalaram quais questões da escala eles acreditavam referir-se ao exercício com um caráter obrigatório ou patológico, sendo que o obrigatório relacionava-se ao bem-estar psicológico dependente da adesão a um regime de exercícios regulares e o patológico, ao exercício que prevalece sobre um componente social da vida do indivíduo. Ao final desse processo, foi elaborada uma nova versão do instrumento ${ }^{16}$.

Para a quarta etapa, essa versão foi apresentada a 116 jovens, de ambos os sexos, com idade média de 24,5 anos (desvio-padrão: 4,7 anos), estudantes do ensino superior do curso de administração. Os jovens concordaram em participar voluntariamente e foram selecionados de forma aleatória simples. Assim, na instituição houve um sorteio para a escolha de três salas e todos os estudantes presentes, em período de aula, responderam a um questionário autoaplicado sob supervisão da autora (MAC). Não houve recusa e/ou desistência. Para uma parcela do grupo (40 jovens), o instrumento foi aplicado na apresentação de uma escala verbal-numérica adaptada ${ }^{26,27} \mathrm{com} o$ intuito de avaliar o grau de compreensão das questões e do instrumento em sua íntegra. Foi utilizado o mesmo procedimento metodológico descrito anteriormente.

Na última etapa, o instrumento foi aplicado ao restante do grupo (76 jovens) na forma de escala Likert de pontos, com a seguinte variação: 1 - nunca, 2 - raramente, 3 - indiferente, 4 - quase sempre, 5 - sempre, para verificar o grau de consistência interna das questões, por meio da análise do coeficiente alfa de Cronbach.

Para as análises estatísticas, utilizou-se o programa SPSS versão 15.0. Desenvolveram-se análises descritiva e inferencial dos dados.

\section{Resultados}

\section{Tradução e adaptação cultural}

$\mathrm{Na}$ tradução do instrumento, houve necessidade de adaptar algumas expressões, conforme descrito no quadro 1 .

$\mathrm{Na}$ tradução do item 3, a frase traduzida como "você tenta compensá-las aumentando o tempo" foi substituída por "você tenta fazê-las colocando em mais tempo de duração", com o intuito de 
oferecer maior compreensão à expressão original. Já para o 5, a expressão "at times when you feel" foi retrotraduzida como "even if you are" e "unwell" foi retrotraduzida como "not well". Dessa forma, optou-se por alterar a tradução de "Você continua a praticar exercícios físicos mesmo quando se sente cansado(a) ou indisposto(a)" para "Você continua a se exercitar nos momentos em que se sente cansado(a) ou indisposto(a)?".

No item 6, a expressão "do you continue to exercise" foi traduzida na primeira versão como "você pratica exercícios físicos mesmo quando", o que levou à retrotradução diferente da versão original. Por isso, optou-se por incluir o verbo "continuar" para enfatizar a pergunta e, dessa forma, garantir a preservação do conteúdo da versão original. Ainda no item 6 , a expressão "have you sustained an exercise-related injury" não obteve retrotradução semelhante, portanto optou-se pela expressão "quando está com o corpo dolorido por causa de lesão relacionada ao próprio exercício físico”.

No item 7, a expressão "let yourself down" foi traduzida como "se sentir triste". Em virtude de a retrotradução não ter sido semelhante, optou-se por traduzi-la como "a ponto de ficar frustrado". Finalmente, no item 8, a expressão "when you turn down" foi traduzida como "você recusa", para tornar mais compreensível à língua portuguesa.

\section{Validação de conteúdo}

Os profissionais $(\mathrm{n}=13)$ analisaram a escala avaliando cada questão sob o aspecto obrigatório e patológico. Para o aspecto obrigatório, que contempla as questões de 1 a 4, ocorreu concordância de 57,7\%; já para o aspecto patológico a concordância correspondeu a 53,9\%, em relação às questões de 5 a 8.

\section{Compreensão verbal}

Considerando as instruções para a utilização da escala e as oito questões, tanto para os especialistas quanto para os estudantes, os valores obtidos foram superiores a 4,0 . Dessa forma, ocorreu um grau de compreensão variando entre 4,3 e 4,9 (máximo 5,0), conforme tabela 1 .

\section{Consistência interna}

O instrumento demonstrou valores do coeficiente alfa de Cronbach aceitáveis (Tabela 1). Para o instrumento, o valor correspondeu a 0,79; já para os aspectos obrigatório (questões de 1 a 4) e patológico (questões de 5 a 8 ), foram de 0,60 e 0,72 , respectivamente.

Quadro 1. Avaliação da equivalência semântica - escala original Commitment Exercise Scale, versão traduzida para o português, retrotraduzida e final do instrumento

\begin{tabular}{|c|c|c|c|}
\hline Original & Tradução & Retrotradução & Final \\
\hline $\begin{array}{l}\text { Instructions: The following are } \\
\text { statements describing attitudes to } \\
\text { exercise. Please respond by marking on } \\
\text { the line beside each statement the point } \\
\text { on the line which best describes your } \\
\text { position on the continuum }\end{array}$ & $\begin{array}{l}\text { Instruções: As frases abaixo descrevem } \\
\text { atitudes quanto ao exercício físico. Por } \\
\text { favor, responda marcando na linha, } \\
\text { correspondente a cada frase, um ponto } \\
\text { que melhor descreve sua posição }\end{array}$ & $\begin{array}{l}\text { Instructions: The phrases below } \\
\text { describe attitudes towards physical } \\
\text { exercise. Please respond by marking the } \\
\text { line corresponding to each phrase on the } \\
\text { point that best describe your opinion }\end{array}$ & $\begin{array}{l}\text { Instruções: As seguintes declarações } \\
\text { descrevem atitudes quanto ao exercício } \\
\text { físico. Por favor, responda, marcando } \\
\text { na linha junto a cada declaração, um } \\
\text { ponto na linha que melhor descreva a } \\
\text { sua posição }\end{array}$ \\
\hline $\begin{array}{l}1 \text { - How important do you think it is to } \\
\text { your general well-being not to miss your } \\
\text { exercise sessions? }\end{array}$ & $\begin{array}{l}1 \text { - } 0 \text { quanto você acha importante para } \\
\text { o seu bem-estar geral não faltar às } \\
\text { aulas de exercício físico? }\end{array}$ & $\begin{array}{l}1 \text { - How important do you think it is for } \\
\text { your general well being not to miss your } \\
\text { exercise sessions? }\end{array}$ & $\begin{array}{l}1 \text { - } 0 \text { quanto você acha importante para } \\
\text { o seu bem-estar geral não faltar às } \\
\text { aulas de exercício físico? }\end{array}$ \\
\hline $\begin{array}{l}2 \text { - Does it upset you if, for one or } \\
\text { another, you are unable to exercise? }\end{array}$ & $\begin{array}{l}2 \text { - Você fica chateado(a) se por algum } \\
\text { motivo você está incapacitado(a) de } \\
\text { praticar exercícios físicos? }\end{array}$ & $\begin{array}{l}2 \text { - Are you uspset if you are unable to } \\
\text { exercise for any reason? }\end{array}$ & $\begin{array}{l}2 \text { - Você fica chateado(a) se por algum } \\
\text { motivo você está incapacitado(a) de } \\
\text { praticar exercícios físicos? }\end{array}$ \\
\hline $\begin{array}{l}3 \text { - If you miss an exercise sessions, or } \\
\text { several sessions, do you try to make } \\
\text { them up by putting in more time when } \\
\text { you get back? }\end{array}$ & $\begin{array}{l}3 \text { - Se você perde uma ou várias } \\
\text { aulas de exercício físico, você tenta } \\
\text { compensá-las aumentando o tempo } \\
\text { quando volta a praticar? }\end{array}$ & $\begin{array}{l}3 \text { - If you miss one or more exercise } \\
\text { sessions, do you try to compensate by } \\
\text { increasing the time when you return? }\end{array}$ & $\begin{array}{l}3 \text { - Se você perder uma ou várias } \\
\text { sessões de exercício físico, você tenta } \\
\text { fazê-las colocando em mais tempo de } \\
\text { duração, quando volta a praticá-lo? }\end{array}$ \\
\hline $\begin{array}{l}4 \text { - Do you have a set routine for your } \\
\text { exercise session e.g. the same time } \\
\text { of day, the same location, the same } \\
\text { number of laps, particular exercises, } \\
\text { and so on? }\end{array}$ & $\begin{array}{l}4 \text { - Você possui uma rotina fixa nas suas } \\
\text { aulas de exercício físico (por exemplo, } \\
\text { na mesma hora do dia, no mesmo lugar, } \\
\text { com o mesmo número de séries, com os } \\
\text { mesmos exercícios etc.)? }\end{array}$ & $\begin{array}{l}4 \text { - Do you have a set routine for your } \\
\text { exercise session (for example, the same } \\
\text { time of day, the same location, the same } \\
\text { number of laps, particular exercises, } \\
\text { and so on)? }\end{array}$ & $\begin{array}{l}4 \text { - Você possui uma rotina fixa nas suas } \\
\text { aulas de exercício físico (por exemplo, } \\
\text { na mesma hora do dia, no mesmo lugar, } \\
\text { com o mesmo número de séries, com os } \\
\text { mesmos exercícios etc.)? }\end{array}$ \\
\hline $\begin{array}{l}5 \text { - Do you continue to exercise at times } \\
\text { when you feel tired or unwell? }\end{array}$ & $\begin{array}{l}5 \text { - Você continua a praticar exercícios } \\
\text { físicos mesmo quando se sente } \\
\text { cansado(a) ou indisposto(a)? }\end{array}$ & $\begin{array}{l}5 \text { - Do you continue exercise even if you } \\
\text { are tired or not well? }\end{array}$ & $\begin{array}{l}5 \text { - Você continua a se exercitar nos } \\
\text { momentos em que se sente cansado(a) } \\
\text { ou indisposto(a)? }\end{array}$ \\
\hline $\begin{array}{l}6 \text { - Do you continue to exercise even } \\
\text { when you have sustained an exercise- } \\
\text { related injury? }\end{array}$ & $\begin{array}{l}6 \text { - Você pratica exercícios físicos } \\
\text { mesmo quando está com o corpo } \\
\text { dolorido por causa dos exercícios } \\
\text { realizados anteriormente? }\end{array}$ & $\begin{array}{l}6 \text { - Do you exercise even when you are } \\
\text { sore from previous exercising? }\end{array}$ & $\begin{array}{l}6 \text { - Você continua a se exercitar mesmo } \\
\text { quando está com o corpo dolorido por } \\
\text { causa de lesão relacionada ao próprio } \\
\text { exercício físico? }\end{array}$ \\
\hline $\begin{array}{l}7 \text { - Do you feel "guilty" that you have to } \\
\text { somehow "let yourself down" when you } \\
\text { miss your exercise session? }\end{array}$ & $\begin{array}{l}7 \text { - Você sente culpado(a) a ponto de se } \\
\text { sentir triste quando falta a uma sessão } \\
\text { de exercício físico? }\end{array}$ & $\begin{array}{l}7 \text { - Do you feel guilty enough to feel sad } \\
\text { when you miss an exercise session? }\end{array}$ & $\begin{array}{l}7 \text { - Você se sente culpado(a) a ponto } \\
\text { de ficar frustrado quando falta a uma } \\
\text { sessão de exercício físico? }\end{array}$ \\
\hline $\begin{array}{l}8 \text { - Are there times when you turn down } \\
\text { an invitation to an interesting social } \\
\text { event because it interferes with your } \\
\text { exercise schedule? }\end{array}$ & $\begin{array}{l}8 \text { - Há situações em que você recusa } \\
\text { convites para eventos sociais porque } \\
\text { acredita que isso interfere na sua rotina } \\
\text { de exercícios físicos? }\end{array}$ & $\begin{array}{l}8 \text { - Do you ever refuse social invitations } \\
\text { because you believe they would } \\
\text { interfere with your exercise routine? }\end{array}$ & $\begin{array}{l}8 \text { - Existem momentos em que você } \\
\text { recusa convites para eventos sociais } \\
\text { interessantes porque isso interfere no } \\
\text { seu horário de exercícios físicos? }\end{array}$ \\
\hline $\begin{array}{l}\text { Not at all important, Very important; } \\
\text { Never upset, Always upset; Never, } \\
\text { Always; No routine, Strict routine; Not } \\
\text { at all, A great deal }\end{array}$ & $\begin{array}{l}\text { Nada importante, Muito importante; } \\
\text { Nunca se chateia, Sempre se chateia; } \\
\text { Nunca, Sempre; Sem rotina, Rotina fixa; } \\
\text { Nem um pouco, Bastante }\end{array}$ & $\begin{array}{l}\text { Not important at all, Very important; } \\
\text { Never upset, Always upset; No routine, } \\
\text { Set routine; Not at all, A lot; Never, } \\
\text { Always }\end{array}$ & $\begin{array}{l}\text { Nada importante, Muito importante; } \\
\text { Nunca se chateia, Sempre se chateia; } \\
\text { Sem rotina, Rotina fixa; Nem um pouco, } \\
\text { Bastante; Nunca, Sempre }\end{array}$ \\
\hline
\end{tabular}


Tabela 1. Avaliação dos especialistas e da população-alvo da Escala de Compromisso ao Exercício Físico

\begin{tabular}{|c|c|c|c|c|}
\hline \multirow[t]{2}{*}{ Questões } & \multicolumn{2}{|c|}{$\begin{array}{l}\text { Especialistas } \\
\qquad(n=13)\end{array}$} & \multicolumn{2}{|c|}{$\begin{array}{l}\text { Estudantes } \\
(n=116)\end{array}$} \\
\hline & $\begin{array}{c}\text { Grau de } \\
\text { concordância } \\
\text { do conteúdo: } \\
\text { aspecto } \\
\text { obrigatório*, } \\
\text { aspecto } \\
\text { patológico** } \\
(\%)\end{array}$ & $\begin{array}{c}\text { Grau de } \\
\text { compreensão } \\
\text { Média (desvio- } \\
\text { padrão) }\end{array}$ & $\begin{array}{c}\text { Grau de } \\
\text { compreensão } \\
\text { Média (desvio- } \\
\text { padrão) } \\
(n=40)\end{array}$ & $\begin{array}{l}\text { Consistência } \\
\text { interna } \\
\alpha \text { de Cronbach } \\
(n=76)\end{array}$ \\
\hline Instrução & - & $4,3(1,0)$ & $4,6(0,9)$ & \\
\hline 1 & 84,6 & $4,6(0,6)$ & $4,6(0,8)$ & \multirow[t]{4}{*}{0,60} \\
\hline 2 & 30,8 & $4,9(0,2)$ & $4,6(0,7)$ & \\
\hline 3 & 38,5 & $4,8(0,3)$ & $4,6(0,6)$ & \\
\hline 4 & 76,9 & $4,7(0,4)$ & $4,5(0,9)$ & \\
\hline 5 & 76,9 & $4,8(0,3)$ & $4,6(0,8)$ & \multirow[t]{4}{*}{0,72} \\
\hline 6 & 76,9 & $4,7(0,4)$ & $4,7(0,8)$ & \\
\hline 7 & 61,5 & $4,7(0,5)$ & $4,7(0,6)$ & \\
\hline 8 & 69,2 & $4,9(0,2)$ & $4,7(0,6)$ & \\
\hline Total & 64,4 & $4,7(0,4)$ & $4,6(0,7)$ & 0,79 \\
\hline
\end{tabular}

${ }^{*}$ Aspecto obrigatório: questões de 1 a 4; ${ }^{* *}$ Aspecto patológico: questões de 5 a 8 .

\section{Discussão}

O presente estudo descreve a etapa inicial da adaptação transcultural da Commitment to Exercise Scale - Escala de Compromisso ao Exercício Físico (ECE). Estudos que zelam pela qualidade do instrumento são essenciais para garantir a qualidade da informação aferida ${ }^{28,29}$.

A escala passou pelo processo de tradução e retrotradução ${ }^{28}$, sendo avaliada por especialistas da área de transtorno alimentar e estudantes. Três versões foram elaboradas até chegar à versão final adaptada.

Para a etapa de tradução e retrotradução, alguns termos não foram traduzidos literalmente, isso porque houve preocupação em sintonizar os conteúdos presentes na versão original com o contexto cultural da população brasileira, pois a correspondência literal de um termo não implica necessariamente a mesma compreensão para distintas culturas ${ }^{30}$. Houve também o cuidado de selecionar vocábulos de fácil compreensão e de abrangência nacional, evitando, assim, os que são utilizados regionalmente ${ }^{31}$.

Dessa forma, em quase todas as questões os pronomes, verbos e conjunções verbais foram adaptados e assim os significados conotativo e denotativo foram considerados na transferência do significado das palavras entre os dois idiomas.

No processo de adaptação, optou-se pela utilização da escala Likert de pontos para facilitar a compreensão dos participantes e a análise dos dados. A versão final do instrumento manteve o formato e a sequência das questões na forma idêntica à proposta na versão original, com exceção da forma de registro da resposta.

A avaliação da compreensão verbal foi excelente (4,3 e 4,9), visto que tanto os profissionais da área quanto os estudantes revelaram quase nenhuma dificuldade para compreender às perguntas contidas no instrumento. Dessa forma, o instrumento comprovou ser de fácil compreensão, não havendo sugestões de nenhum dos participantes para alterações estruturais. Sendo assim, os componentes da versão original foram mantidos nas versões traduzida e adaptada. Entretanto, na análise de conteúdo, realizada pelos profissionais, observaram-se percentuais de grau de concordância nas questões 2 e 3 (referentes ao aspecto obrigatório) baixos. Isso significa que os profissionais relacionaram tais questões com o aspecto patológico. Esse fato deu-se provavelmente pelas questões não serem tão claras em relação aos conteúdos que a contemplam. Para responder a essa questão, faz-se necessária a realização de análise fatorial. Mesmo assim, para os conteúdos "aspecto obrigatório" e "aspecto patológico", houve concordância de $60 \%$ e $71 \%$, conforme descrito na tabela 1 .
$\mathrm{Na}$ análise de consistência interna, o instrumento apresentou valor correspondente a 0,79 , similar ao encontrado na versão original $(0,77)$. Já em relação aos construtos “aspecto obrigatório" e "aspecto patológico”, os valores corresponderam a 0,60 e 0,72 , respectivamente. Dessa forma, observa-se que o instrumento respondeu de forma satisfatória, preservando a coerência e a integração dos itens que o compõem, bem como em relação aos construtos.

Vale ressaltar que as estratégias para o processo de adaptação transcultural de instrumentos para pesquisa são inúmeras ${ }^{31-33}$. É de suma importância que as etapas de operacionalização do processo sejam rigorosamente descritas, para assim garantir a veracidade e a qualidade da variável pesquisada. Em estudos epidemiológicos, torna-se fundamental zelar pela qualidade da informação, haja vista o elo existente entre o conteúdo teórico e o dado empírico que expressa essa realidade $28-30$.

A falta de escalas brasileiras validadas para avaliar a relação entre exercício físico e transtornos alimentares é uma das fragilidades do processo de validação do ECE. A não existência de dados publicados não permite comparação com os achados do presente estudo. Rosa et al. ${ }^{34}$ publicaram uma versão brasileira do Negative Addiction Scale ${ }^{9}$, traduzida como Escala de Dependência ao Exercício ${ }^{34}$, que avalia os aspectos da dependência de exercício físico relacionada com o histórico de corrida do indivíduo. Apesar de ter apresentado valores de correlação significativos, as questões avaliam exclusivamente o hábito de correr em atletas profissionais e amadores. Segundo De Coverley Veale ${ }^{9}$, esse tipo de dependência poderia ser definida como primária, por se tratar de uma prática com o único fim de se exercitar, portanto deve ser excluído o diagnóstico de transtorno alimentar.

O ECE tem por objetivo avaliar o exercício físico como método de purgação ou compensação em indivíduos com transtornos alimentares, uma vez que o exercício físico pode ser utilizado por homens e mulheres que se preocupam excessivamente com o peso e a autoimagem.

Os impactos positivos da prática regular de exercício físico na saúde e na qualidade de vida das pessoas são bem conhecidos. No entanto, indivíduos com transtornos alimentares frequentemente não se beneficiam, na medida em que podem utilizá-los como estratégia para perder peso, de forma inadequada e por vezes de forma compulsiva ou excessiva. Estudos do comportamento de indivíduos com transtornos alimentares quanto à prática de exercícios físicos são escassos em nosso país. O uso de escalas como essa possibilita avaliar se a relação do indivíduo com o exercício físico pode ser considerado obsessivo e/ou patológico, considerando aspectos como comprometimento, dependência e frequência desse comportamento.

Conclui-se, a partir do processo de adaptação transcultural e pelos resultados satisfatórios encontrados, que a versão brasileira da escala (Anexo 1) está em condições de ser recomendada para futuras aplicações. Ainda são necessárias análises de validação de construto e fatorial, bem como avaliação de sua reprodutibilidade. Sendo assim, é possível afirmar que o presente instrumento será útil para futuras análises psicométricas, o que possibilitará futuramente sua aplicação e avaliação da prática de exercícios físicos em pacientes com transtornos alimentares.

\section{Referências}

1. Blair SN, Kohl HW, Paffenbarger RS, Clark DG, Cooper KH, Gibbons LW. Physical fitness and all-cause mortality. JAMA. 1989;262:2395-401.

2. Matsudo SMM, Araujo T, Matsudo VR, Andrade D, Andrade E, Oliveira L, et al. Questionário Internacional de Atividade Física (IPAQ): estudo de validade e reprodutibilidade no Brasil. Rev Bras Ativ Fís Saúde. 2001;6:5-18.

3. Matsudo SMM, Matsudo VR, Araújo T, Andrade D, Andrade E, Oliveira L, et al. Nível de atividade física da população do Estado de São Paulo: análise de acordo com o gênero, idade, nível socioeconômico, distribuição geográfica e de conhecimento. Rev Bras Ciên Mov. 2002;10:41-50.

4. Haskell WL, Lee I-Min, Pate RR, Powell KE, Blair SN, Franklin BA, et al. Physical activity and public health. Circulation. 2007;116:1081-93. 
5. Pardini R, Matsudo SM, Araújo T, Matsudo V, Andrade E, Braggion G, et al. Validação do questionário internacional de nível de atividade física (IPAQ - versão 6): estudo piloto em adultos jovens brasileiros. Rev Bras Ciên Mov. 2001;9:45-51.

6. Ogden J, Veale D, Summers Z. The development and validation of the Exercise Dependence Questionnaire. Addict Res. 1997;5:343-56.

7. Hausenblas HA, Cook BJ, Chittester NI. Can exercise treat eating disorders? Exerc Spor Sci Rev. 2008;36:43-7.

8. Pierce EF. Exercise dependence syndrome in runners. Sports Med. 1994;18:149-55.

9. De Coverley Veale DME. Exercise dependence. Br J Addict. 1987;82:735-40.

10. Hailey BJ, Bailey LA. Negative addiction in runners: a quantitative approach. J Sport Behav. 1982;5:150-4.

11. Morgan WP. Negative addiction in runners. Phys Sportsmed. 1979;7:57-77.

12. Coen SP, Ogles BM. Psychological characteristics of the obligatory runner: a critical examination of the anorexia analogue hypothesis. J Sport Exerc Psychol. 1993;15:338-54.

13. Hausenblas HA, Dows DS. How much is too much? The development and validation of the exercise dependence scale. Psychol Health. 2002;17:387-404.

14. Veale D. Does primary exercise dependence really exist? In: Annett J, Cripps B, Steinberg H. Exercise addiction: motivation for participation in sport and exercise (Leicester, UK): British Psychological Society; 1995, p. 1-5.

15. Pasman L, Thompson JK. Body image and eating disturbance in obligatory runners, obligatory weightlifters, and sedentary individuals. Int J Eat Disord. 1988;7:759-69.

16. Davis C, Brewer H, Ratusny D. Behavioral frequency and psychological commitment: necessary concepts in the study of excessive exercising. J Behav Med. 1993;16:611-28.

17. American Psychiatric Association (APA). Diagnostic and Statistical Manual of Mental Disorders. 4. ed. Washington DC; 1994.

18. Davis C, Kaptein S. Anorexia nervosa with excessive exercise: a phenotype with close links to obsessive-compulsive disorder. Psychiatry Res. 2006;142:209-17.

19. Shapiro JR, Berkman ND, Brownley KA, Sedway JA, Lohr KN, Bulik CM. Bulimia nervosa treatment: a systematic review of randomized controlled trials. Int J Eat Disord. 2007;40:321-36.

20. Peñas-Lledó E, Leal FJV, Waller G. Excessive exercise in anorexia nervosa and bulimia nervosa. Int J Eat Disord. 2002;31:370-5.

21. Hoek H, Van Hoeken D. Review of the prevalence and incidence of eating disorders. Int J Eat Disord. 2003;34:383-96.
22. Adkins EC, Keel PK. Does "excessive" or "compulsive" best describe exercise as a symptom of bulimia nervosa? Int J Eat Disord. 2005;38:24-9.

23. Carmack MA, Martens R. Measuring commitment to running a survey of runners' attitudes and mental states. J Sport Psychol. 1979;1:25-42.

24. Mond JM, Hay PJ, Rodgers B, Owen C. An update on the definition of "excessive exercise" in eating disorders research. Int J Eat Disord. 2006;39:147-53.

25. Reichenheim ME, Moraes CL. Operacionalização de adaptação transcultural de instrumentos de aferição usados em epidemiologia. Rev Saude Publica. 2007;41:665-73.

26. Conti MA, Latorre MRDO, Hearst N, Segurado A. Cross-cultural adaptation, validation and reliability of the Body Area Scale for Brazilian adolescents. Cad Saude Publica. 2009;25:2179-86.

27. Conti MA, Slater B, Latorre MRDO. Validação e reprodutibilidade da Escala de Evaluación da Insatisfación Corporal para Adolescentes. Rev Saude Publica. 2009;43:515-24.

28. Gullemin F, Bombardier C, Beaton D. Cross-cultural adaptation of health related quality of life measures: literature review and proposed guidelines. J Clin Epidemiol. 1993;46(12):1417-32.

29. Beaton DE, Bombardier C, Guillemin F, Ferraz MB. Guidelines for the process of cross-cultural adaptation of self-report measures. Spine. 2000;25(24):3186-91.

30. Reichenheim ME, Paixão Jr CM, Moraes CL. Adaptação transcultural para o português (Brasil) do instrumento Hwalek-Sengstock Elder Abuse Screening Test (H-S/EAST) utilizado para identificar risco de violência contra o idoso. Cad Saude Publica. 2008;24:1801-13.

31. Mattos P, Segenreich, Saboya E, Louzã M, Dias G, Romano M. Adaptação transcultural para o português da escala Adult Self-Report Scale para avaliação de transtorno de déficit de atenção/hiperatividade (TDAH) em adultos. Rev Psiquiatr Clín. 2006;33(4):188-94.

32. Scagliusi FB, Cordas TA, Polacow VO, Coelho D, Alvarenga M, Phillipi ST, Lancha Jr AH. Tradução da escala de desejo de aceitação social de Marlowe e Crowne para a língua portuguesa. Rev Psiquiatr Clín. 2004;31(6):272-8.

33. Brietzke E, Daruy Filho L, Grassi-Oliveira R. "Life Chart" Retrospectivo: instrumento para assinalar graficamente a presença e a evolução do impacto funcional de episódios afetivos maníacos e depressivos. Rev Psiquiatr Clín. 2009;36(6):217-20.

34. Rosa DA, Mello MT, Souza-Formigoni MLO. Dependência da prática de exercícios físicos: estudo com maratonistas brasileiros. Rev Bras Med Esporte. 2003;9:9-14.

\section{Anexo 1}

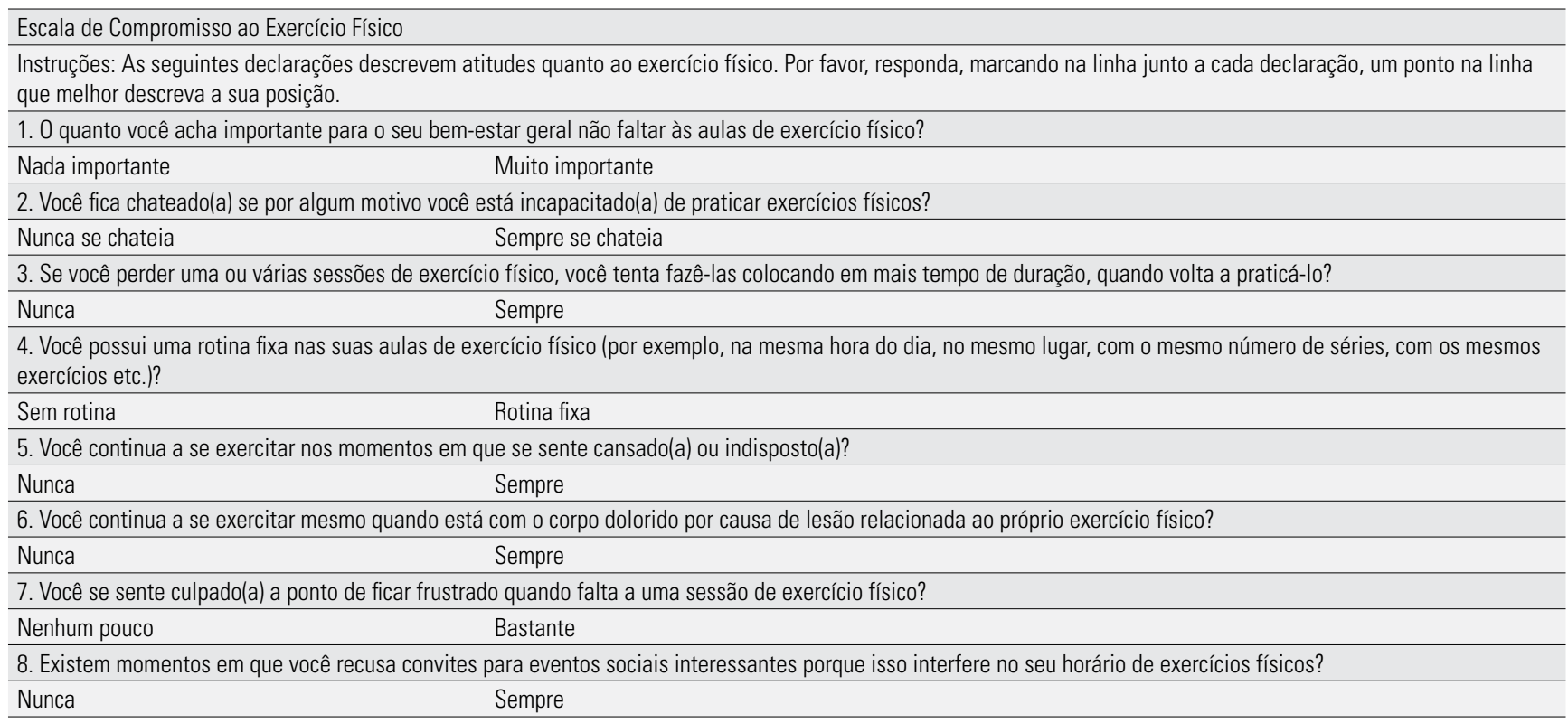

